\title{
As crises de cefaleia podem ter duração realmente menor que duas horas na infância e na adolescência? Análise crítica prospectiva dos critérios diagnósticos da ICHD II
}

\author{
Márcia M. F. Lima', Luis C. Martin² \\ 'Professora Assistente da Disciplina de Neurologia Infantil e Responsável pelo Ambulatório de \\ Cefaleias na Infância e na Adolescência do HC da FM da UNESP Botucatu \\ ${ }^{2}$ Professor Dr. Clínica Médica - HC Botucatu, UNESP - Assessoria estatística \\ Lima MF, Martin LC. As crises de cefaléia podem ter duração realmente menor que duas horas na \\ infância e na adolescência?Análise crítica prospectiva dos critérios diagnósticos da ICHD II. \\ Headache Medicine. 2012;3(4):207-8
}

\section{INTRODUÇÃO}

Os critérios diagnósticos da ICHD foram revistos, o que resultou em algumas mudanças na ICHD II,') como reconhecer que a duração das crises de migrânea poderiam durar de uma a 72 horas.

Alguns autores observaram duração menor que duas horas (11\%-81\%) e menos que uma hora $(8 \%$ 25\%).

Winner et al. ${ }^{(2)}$ referiram que esta redução aumentaria a sensibilidade de $66 \%$ para $78 \%$ usando o critério clínico diagnóstico como gold standard".

Maytal et al.(3) demonstraram que, diminuindo a duração para menos de uma hora, haveria poucos ganhos e redução na especificidade.

Em relação à validação da ICHD II continua a ser testada, Lima et al.(4) observaram que a sensibilidade para o diagnóstico da migrânea sem aura aumentou de $21 \%$ para $53 \%$ e da migrânea com aura de $27 \%$ para $71 \%$ sem alteração da especificidade.

Um estudo da American Headache Society Pediatric-Adolescent Section ${ }^{(5)}$ demonstrou que com a diminuição da duração da dor os critérios são diagnosticados em $80,8 \%$.

\section{OBJETIVO}

Analisar criticamente os critérios da Classificação Internacional das Cefaleias (ICHD II)-2004 prospectivamente, visto que, na classificação, há uma proposição que esta verificação se faça para termos uma maior validação e evidência científica. 


\section{MÉTODO}

Analisamos prospectivamente 150 indivíduos de Serviço ambulatorial, não tratados, orientando o preenchimento do diário da dor durante a primeira consulta, com retorno após 30 dias. Observamos todos os critérios da classificação ICHD II com crises $>2$ horas (Grupo ICHD II) e os mesmos critérios, mas sendo que o critério duração das crises em crianças seria menor que 2 horas (Grupo ICHD II-P). Empregou-se o critério diagnóstico clínico intuitivo (DCl) como padrão gold standard. A comparação entre CDI, ICHDII, ICHDII - P foi realizada através do teste de Mc Nemar.

\section{RESULTADOS}

Idade de $11 \pm 2$ anos, 34\% sexo masculino/66\% sexo feminino; em relação aos sintomas: náusea $-65 \%$, vômitos - 52\%, fotofobia-96\%, fonofobia - 97\%, tontura - 52\%, osmofobia - 54\%, sendo que este último não acrescenta ao diagnóstico, pois todos que a apresentavam também tinham foto/fonofobia. Duração: ICHD II 88 crises $>2$ horas e ICHD II-P - 62 crises $<2$ horas.

Observamos que os critérios da ICHD II apresentam uma sensibilidade (S) de $58 \%$ quando comparadas aos critérios do CDI - 100\% e os da ICHDII-P - 94\% com Mc Nemar $p<0,001$.

\section{CONCLUSÕES}

O uso dos critérios atuais da ICHD II, através da evidência de um estudo prospectivo demonstra um aumento importante (94\%) da sensibilidade comparada a $(S=58 \%)$ da duração (> 2horas). Podemos então aceitar a duração $<2$ horas para as cefaleias na infância e na adolescência.

\section{REFERÊNCIAS}

1. Headache Classification Sub-Committee of the International Headache Society. The International Classification of Headache Disorder, $2^{\text {nd }}$ ed. Cephalalgia. 2004;24:1 - 160

2. Winner P, Martinez W, Mate L, Bello L. Classification of pediatric migraine: proposed revisions to the IHS criteria. Headache. 1995;35(7):407-10

3. Maytal J, Young M, Shechter A, Lipton RB. Pediatric migraine and the International Headache Society (IHS) criteria. Neurology. 1997;48(3):602-7

4. Lima MM, Padula NA, Santos LC, Oliveira LD, Agapejev S, Padovani C. Critical analysis of the international classification of headache disorders diagnostic criteria (ICHD I-1988) and (ICHD II-2004), for migraine in children and adolescents. Cephalalgia. 2005;25 (1 1):1042-7.

5. Hershey AD, Winner P, Kabbouche MA, Gladstein J, Yonker M, Lewis $D$, et al. Use of the ICHD-II criteria in the diagnosis of pediatric migraine. Headache. 2005;45(10):1288-97. 